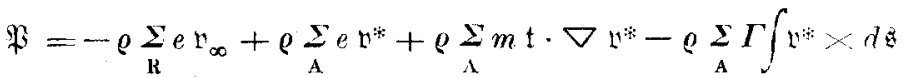

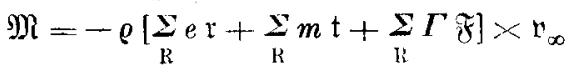

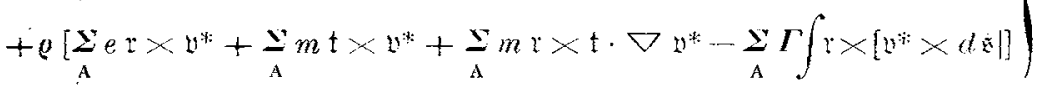

Durch eine ähnliche Umformung wie in (23') $\mathrm{kann}$ man noch $-\Sigma \Gamma \widetilde{F} \times \mathrm{v}_{\infty}$ durch $\Sigma \Gamma \int \mathfrak{r} \times\left[r_{\infty} \times d \xi\right]$ ersetzen und damit die formale Aehnlichkeit der Zusatzglieder mit den bei der inneren Aufgabe auftretenden Gliedern auch für die Wirbellinien herstellen.

11. Schlufbemerkung. Die Bebandlung der entsprecheaden ebenen Aufgabe führt zwar, am besten mit funktionentheoretischen Hilfsmitteln, zu ähnlichen Ergebnissen wie im Raum; indessen können diese nicht in einfacher Weise ans den hier abgeleiteten Sätzen durch Grenzïbergang erhalten werden. Ein wesentlicher Unterschied liegt darin, daß in der Ebene Wirbelpunkte, die den Stabwirbeln im Raum entsprechen, nicht ausgeschlossen zu werden brauchen; im Gegenteil sind sie von größtem und leicht berechenbarem Einfluß auf $\mathrm{Kraft}^{1}$ ) und Moment. Die entstehenden Formeln enthalten als einfachste Fälle die Kutta-Jou kowskische Gleichung und die Blasiasschen Formeln, deren Gültigkeitsbereich sie erheblich erweitern.

171

\title{
Potentialströmung durch Gitter. $\left.{ }^{2}\right)$
}

\author{
Von E. KŐNIG in Dresden.
}

$\mathrm{D}$ e Erfolge der Potentialtheorie in der Aerodyoamik legen es nahe, diese Theorie auch auf Fragen der Turbinenlehre anzuwenden. Dabei hat man es mit der Strömung durch die Reihe der Turbinenkanäle zu tun, die man als ein regelmäBiges "Gitter" idealisieren kann. Die folgende Abhandlung bat zum Ziele, besondere Fragen, die bei Strömung durch Gitter auftreten, unter den Voraussetzungen, die die Potentialtheorie für das zweidimensionale Gebiet macht, zu diskutieren.

1. Die Joukowskische Theorie und ihre Erweiterung auf Gitter. Die Joukowskische Theorie geht bekanntlich aus von der konformen Abbildung des Strömungsgebietes in der Umgebung einer ebenen Platte auf das Gebiet im Außenraum eines Kreiszylinders. Von der Platte und dem Zylinder kommen dabei nur die ebenen Schnitte, Gerade bezw. Kreis, in Betracht. Statt der Abbildung auf das Aeußere des Kreises kann man auch die aut die Halbebene nebmen. Um die Analogie zwischen der Joukowskischen Theorie und der hier angewandten Methode hervortreten zu lassen, stellen wir die Joukowskische Theorie folgendermaßen dar: Der Schnitt einer ebenen Platte mit einer $z$-Ebene senkrecht zur Platte sei gegeben durch die Strecke $A B$, die gegen die negative $x$-Achse unter dem Winkel $\alpha$ geneigt ist. Nun richten wir die Jonkowskische Abbildung so ein; daß die $z$-Ebene außerhalb der Strecke $A B$ auf die Halbebene $\eta>0$ einer $\zeta$-Ebene abgebildet wird. Dabei soll die reelle Achse der $\zeta$-Ebene das Bild von $A B$ werden. Die Abbildungsfunktion lautet:

$$
\frac{z-A}{z-B}=e^{-i \pi \alpha \zeta^{2}}
$$

In $A$ und $B$ der $z$-Ebene liegen Vorderkante und Hinterkante des Profils. Die Vorderkante $A$ geht in den Punkt $\zeta=0$ über, die Hinterkante $B$ in den Punkt $\zeta=\infty$. Der Punkt $z=\infty$ wird $\zeta=i$. Der Oberseite von $A B$ entspricht der positive Halbstrabl der $\xi$-Achse und der Unterseite von $A B$ der negative Halbstrahl der $\xi$-Achse. In dieser Darstellung läbt sich die Joukowskische Theorie auch auf kreisbogenförmige Profile, dicke Profile und an den Enden zugespitzte Profile anwenden ${ }^{3}$ ).

1) Vergl, die S, 409 unter (1) zitierte Abbandlung S, 221 bis 224.

2) Auszug aus einer von ỏer phliosophischen Fakultät der Universitut Gätingen angenommenen Dissertation.

${ }^{3}$ ) Vergl. v. Kármán-Treff $\mathbf{z}$ : Potentialströmung um gegebene Tragflachenquerschnitte. Ztschr. f. Flugtechnik u. Motor-Luftschiffahrt, 1918 
2. Die Abbildung. Es ist nun unsere Aufgabe, diese Methode für ein Gitter mit unendlich vielen Schaufeln auszubilden. Es sei ein Gitter von unendlich vielen, geradlinigen, unendlich dünnen Konturen (Schaufelo) und "geneigter Gitterachse«, die die Enden der Schaufel verbindet, gegeben (Abb. 1). Führen wir ein Koordinatensystem so ein, daß die horizontal liegenden Schanfeln die Richtung für die $x$-Achse geben, und eine von ihnen auf der $x$-Achse liegt; ferner, das die Gitterachse mit der positiven Richtung der $x$-Achse einen Winkel $\frac{\pi}{2}+\alpha$ bildet, die Gitterachse gegen die $y$-Achse also um den Winkel $\alpha$ geneigt ist. Der Abstand zweier Schaufeln längs der Gitterachse sei $t$. Legen wir durch die Schaufeln parallel der $x$-Achse Geraden, so wird die ganze $x-y$-Ebene in parallele Streifen aufgeteilt.

Ist nun in dieser Ebene, die als $z$-Ebene bezeichnet wird, ein Punkt $z_{0}=x_{0}+i y_{0}$ gegeben, so gelangt man zu dem kongruenten Punkte im benachbarten Streifen, wenn man von $z_{0}$ zu $z_{0}+i t e^{i \alpha}$ geht, d. h. in der $x$-Richtung um das Stuick $t$ sin $\alpha$ nach links und in der $y$-Richtung um $t \cos \alpha$ nach oben fortschreitet. Ein solcher Streifen von der Breite $t \cos \alpha$ stellt also für $z=x+i y$ einen $\mathrm{Pe}-$ riodenstreifen dar.

Wird ein solcher Periodenstreifen der $z$-Ebene auf eine $\zeta$-Ebene konform durch

$$
\zeta^{*}=e^{2 \pi e^{-i \alpha} \frac{z}{t}}
$$

abgebildet, so überdeckt die Abbildung der übrigen Periodenstreifen jedesmal die $\zeta^{*}$-Ebene in gleicher Weise, d. b. so, daß kongruente Punkte zu demselben $\zeta$ - Werte gehören; somit ergibt sich folgende Abbildung der $z$-Ebene auf die unendlich vielblätrige $\zeta *$ Ebene. Es entspricht $\zeta^{*}=0$ dem Punkte $z=-\infty$ und $\zeta^{*}=+\infty$ dem Punkte $z=+\infty$. Geht man nun von einem Punkte $z$ zu dem kongruenten Pankte $z+i t e^{i a}$, so wird

$$
\zeta^{*}\left(z+i t e^{i \alpha}\right)=\zeta^{*}(z) e^{2 \pi i}=\zeta^{*}(z)
$$

Um die Abbildung auf die $\zeta^{*}$-Ebene zu übersehen, benutzen wir die logarithmische Schreibweise:

$$
\ln \zeta^{*}=2 \pi \frac{z}{t}(\cos \alpha-i \sin \alpha) .
$$

Bekommt der Realteil oder Imaginärteil von $\ln \zeta^{*}$ einen konstanten Wert, so entsprechen ihm in der $z$-Ebene Geraden parallel und senkrecht zur Gitterachse. In der $\zeta^{*}$-Ebene entsprechen dem Realteil Kreise um den Nullpunkt und dem Imaginarteil Geraden durch den Nullpunkt. Nun war in der $z$-Ebene das Koordinatensystem so gewählt, daß die als geradlinig vorausgesetzten Sohaufeln in Richtung der $x$-Achse fallen. Diese schneiden aber die Geraden, die $\Re\left({ }^{\prime} \zeta^{*}\right)=$ konst. entsprechen, unter konstantem Winkel. Also wird in der $\zeta^{*}$-Ebene die Schanfel sich auf ein Kurvenstück abbilden, das die Radien durch den Nullpankt bezw. die Kreise um den Nullpunkt unter konstantem Winkel schneidet, also auf ein Stück einer logarithmischen Spirale.

In der $\zeta^{*}$.Ebene haben wir also folgende Abbildung der $z$-Ebene, die wir der Anschanlichkeit wegen als Strömung beschreiben, d. h. wir fassen die Bilder der Geraden $y=$ konst. als Stromlinien, die Graden $x=$ konst. als Potentiallinie einer Potentialströmung in der $\zeta^{*}$-Ebene auf, and zwar geht die Strömung in Iogarithmischen Spiralen von der Quelle $\zeta^{*}=0$ zur Senke $\zeta^{*}=\infty$. Ebenso sind die Bilder der Potentiallinien $x=$ konst. logarithmische Spiralen, die auf den logarithmischen Spiralen $y=$ konst senkrecht steben. Beide kommen aus dem Punkte $\zeta^{*}=0$ heraus, und zwar schneiden die Linien $y=$ konst. die Geraden durch den Nullpunkt unter dem Winkel $\alpha$. Wir erhalten also eine Strömung, bei der im Nullpunkte eine Quelle von der Ergiebigkeit $\Re\left(\ln \zeta^{*}\right)$ und ein Wirbelfaden mit der Zirkulation $\Im\left(\ln \zeta^{*}\right)$ liegt. Dieses Zusammenfallen von Quelle und Wirbelfaden bezeichnen wir kurz als "Wirbelquelle«. Natürlich liegt dem entsprechend in dem unendlich fernen Pankte eine "Wirbelsenke". Wir bezeichnen das Bild der Schaufel (d. h. das Stück der logarithmischen Spirale $y=0$ ) mit $H-V$ ( $H$ Hinterkante, $V$ Vorderkante). Jetzt bilden wir weiter das Gebiet um das 
Schaufelbild in der $\zeta *$-Ebene auf die obere $\zeta$-Halbebene ab $(\zeta=\xi+i \eta)$. Bei dieser $\mathrm{Ab}$ bildung möge die Wirbelquelle und die Wirbelsenke in die Pankte $\zeta=\zeta_{0}$ und $\zeta=\zeta_{1}$ rücken. Die Sehanfel gehe in die $\xi$-Achse über und zwar die Vorderkante in den Nullpunkt und die Hinterkante in das Unendlichferne. Diese Abbildung erreicht man offenbar dadurch, dab man Wirbelquelle und Wirbelsenke an der $\xi$-Achse spiegelt, wodurch diese Stromlinie wird. Wir können nun in einfacher Weise die Beziehung zwischen der $z$-Ebene und der $\zeta$-Ebene herstellen. Betrachten wir $z$ als Funktion von $\zeta$, so muß nach dem Vorhergehenden zunächst an den Stellen $\zeta=\zeta_{0}$ und $\zeta=\zeta_{1}$ eine Wirbolquelle bezw. eine Wirbelsenke liegen. In der unteren Hälfte der $\zeta$-Ebene liegen die Spiegelbilder der Wirbelquelle und der Wirbelsenke in $\bar{\zeta}_{0}$ und $\bar{\zeta}_{1}$.

Die Wirbelquelle and Wirbelsenke wird dargestellt durch die Funktion

$$
C e^{i \alpha} \ln \frac{\zeta-\zeta_{0}}{\zeta-\zeta_{1}}
$$

Entsprechend der Spiegelung fügen wir die konjugierte Funktion hinzn und erhalten:

$$
z=C\left\{e^{i \alpha} \ln \frac{\zeta-\zeta_{0}}{\zeta-\zeta_{1}}+e^{-i \alpha} \ln \frac{\zeta-\bar{\zeta}_{0}}{\zeta-\bar{\zeta}_{1}}\right\} .
$$

3. Beziehung zwischen den geometrischen Gröfen der Schaufelanordnung und den Konstanten der Abbildungsfunktion. Wir wollen nun die Beziehungen zwischen den geometrischen Größen der Schaufelanordnung und den Konstanten $\zeta_{0}$ und $\zeta_{1}$ herstellen. Dem Punkte $\zeta=\zeta_{0}$ entspricht $z=-\infty$ (Abb. 2) und $\zeta=\zeta_{1}$ der Pankt $z=+\infty$ Die Ableitung der Abbildungsfunktion

$$
\frac{a z}{d \zeta}=C\left\{e^{i \alpha} \frac{\left(\zeta_{0}-\zeta_{1}\right)}{\left(\zeta-\zeta_{0}\right)\left(\zeta-\zeta_{1}\right)}+e^{-i \alpha} \frac{\left(\overline{\zeta_{0}}-\overline{\zeta_{1}}\right)}{\left(\zeta-\overline{\zeta_{0}}\right)\left(\zeta-\bar{\zeta}_{1}\right)}\right\} \cdots . .
$$

besteht ebenfalls, wie die Abbildungsfunktion selber, aus der Summe zweier konjugiertkomplexer Größen. Für reelle Werte von $\zeta$ wird also auch $\frac{d z}{d \zeta}$ reell, und somit ist das

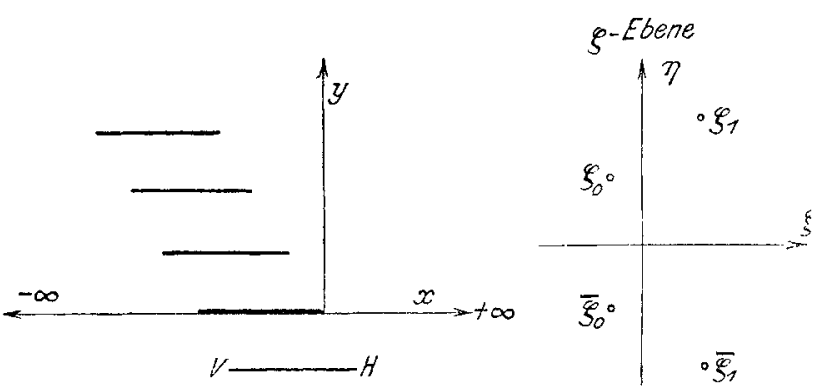

Abb. 2
Bild der $\xi$-Achse horizontal. Der Ausdruck (4) verschwindet für zwei Werte von $\zeta$. Wir erhalten also zwei Verzweigungspunkte, die der Vorderkante $V$ und der Hinterkante $H$ der Schanfel entsprechen, und wir wollen verlangen, daß der eine in den Nullpunkt fällt, der andere in das Unendlichferne. Diese Bedingungen bestimmen $\zeta_{0}$ und $\zeta_{1}$. Für $\zeta=0$ soll also $\frac{d z}{d \zeta}=0$ sein,

und für $\zeta=\infty$ mub $\frac{d z}{d \zeta}$ von der dritten Ordnung verschwinden. Schreiben wir die Gl. (4) in der Form:

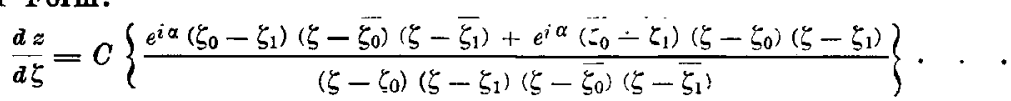

so muß im Zähler das Glied mit $\zeta^{2}$ versohwinden, damit die Bedingung im Unendlichen erfüllt ist, und das absolute Glied muB gleich Null werden, damit $\zeta=0$ Verzweigungspunkt wird, d. h. es mub

$$
\begin{gathered}
e^{i a}\left(\zeta_{0}-\zeta_{1}\right)+e^{-a}\left(\bar{\zeta}_{0}-\bar{\zeta}_{1}\right)=0 \\
e^{i a}\left(\zeta_{0}-\zeta_{1}\right) \bar{\zeta}_{0} \bar{\zeta}_{1}+e^{-i \alpha}\left(\bar{\zeta}_{0}-\bar{\zeta}_{1}\right) \zeta_{0} \zeta_{1}=0
\end{gathered}
$$

und

sein. Setzen wir (Abb. 3)

so wird nach Gl. (6)

$$
\zeta_{0}-\zeta_{1}=d e^{i \delta} \quad, \zeta_{0}-\zeta_{1}=d e^{-i \delta}
$$

$$
\cos (\alpha+\delta)=0 \text { oder } \alpha+\delta=1 / 2 \pi \text {. }
$$

Setzen wir ferner $\zeta_{0}=r_{0} e^{i \vartheta_{0}}, \zeta_{1}=r_{1} e^{i \vartheta_{1}}, \bar{\zeta}_{0}=r_{0} e^{-i \vartheta_{0}}, \bar{\zeta}_{1}=r_{1} e^{-i \vartheta_{1}}$, so folgt aus GI. (7) bei Beri:cksichtigung von (8)

$$
\sin \left(\boldsymbol{\vartheta}_{0}+\boldsymbol{\vartheta}_{1}\right)=0 \text { und somit } \boldsymbol{\vartheta}_{0}+\vartheta_{1}=\pi
$$


G]. (5) erhält dann die Form:

$$
\frac{d z}{d \zeta}=-C d^{2} \frac{2 \zeta \cos \alpha}{\left(\zeta-\zeta_{0}\right)\left(\zeta-\zeta_{1}\right)\left(\zeta-\bar{\zeta}_{0}\right)\left(\zeta-\zeta_{1}\right)}
$$

Die Punkte $\zeta_{0}$ und $\zeta_{1}$ miüssen also, wenn die Verzweigungspunkte (Schaufelenden) in den Nullpunkt nnd das Unendlichferne fallen, anf zwei Halbstrahlen liegen, die symmetrisch zur $\eta$-Richtung liegen. Die Lage der Punkte $\zeta_{0}$ und $\zeta_{1}$ auf diesen Halbstrahlen ist nach Gl. (8) so zu wählen, daß ihre Verbindungsstrecke den Winkel $\delta=\pi / 2-x$ mit der $\xi$-Achse bildet. Wegen der Willkürlichkeit des Maßstabes in der $\zeta$-Ebene (Gl. (3) ist homogen und vom Grade Null in $\zeta$ ) ist die Gerade nur der Richtung nach bestimmt, ihre Lage ist beliebig.

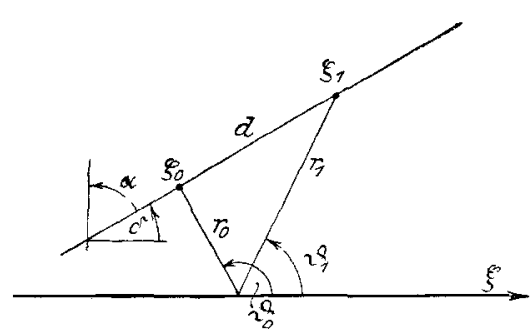

Abb. 3

Durch die Abbildung geht nun der Pankt $\zeta=\infty$ in den Punkt $z=0$ ïber, dadurch ist in der $z$-Ebene der Nullpunkt des Koordinatensystems bestimmt. Dem Punkt $\zeta=0$ entspricht dann die Vorderkante

$$
z_{0}=2 C\left\{\cos \alpha \ln \frac{r_{0}}{r_{1}}-\left(\vartheta_{0}-\vartheta_{1}\right) \sin \alpha\right\} \text {. . . . . . }
$$

$z_{0}$ ist negativ, wie wir sehen werden, entsprechend der Festsetzang, dab die Vorderkante links von der Hinterkante liegen soll (siehe Abb. 2). Da die Hinterkante im Nullpunkt liegt, gibt der Absolutbetrag von $z_{0}$ die Schaufellänge $l$ an.

Wird ferner in der $\zeta$-Ebene von einem beliebigen Punkte der $\xi$-Achse aus ein geschlossener Weg durchlaufen, z. B. einmal um den Punkt $\zeta_{0}$ herum, so daß der Punkt $\zeta_{0}$ danernd zur Linken bleibt, und wird über diesen Weg integriert, so wird

$$
\int_{\zeta} d z=C e^{i a} 2 \pi i \text {. }
$$

In dem Bilde dieses Weges in der $z$-Ebone kehren wir nicht zum Ausgangspunkt zurüok, sondern kommen zu dem kongruenten Punkte der nächsten Schaufel. Ein Umlauf von $\zeta=0$ um $\zeta=\zeta_{0}$ berum nach $\zeta=0$ zarück ergibt also in der $z$-Ebene einen Weg von einer Schaufelvorderkante zur nächsten. Der Imaginärteil von $C e^{i a} \cdot 2 \pi i$ liefert den Abstand der Schaufeln

und der Realteil die Staffelung

$$
h=2 \pi C \cos \alpha
$$

$$
p=-2 \pi C \sin \alpha .
$$

Es fragt sich nun, wie wir $\zeta_{0}$ und $\zeta_{1}$ zu wählen haben, um ein vorgegebenes Gitter abzubilden. Aus der Zasammenfassung von Gl. (10) und (11) ergibt sich: .

$$
\pi \underset{h}{\stackrel{l}{l}=\ln }{ }_{r_{1}}^{r_{0}}-\left(\vartheta_{0}-\vartheta_{1}\right) \text { tang } \alpha
$$

Berücksichtigt man, daß $\vartheta_{0}=\pi-\vartheta_{1}$ ist and

$$
\frac{r_{0}}{r_{1}}=\frac{\sin \left(\vartheta_{1}-\delta\right)}{\sin \left(\vartheta_{1}+\bar{\delta}\right)}=-\frac{\cos \left(\vartheta_{1}+\alpha\right)}{\cos \left(\vartheta_{1}-\alpha\right)}
$$

wie es aus Abb. 3 abzulesen ist, so folgt

$$
\operatorname{tang} \vartheta_{1}=\frac{1+\frac{r_{0}}{r_{1}}}{1-\frac{r_{0}}{r_{1}}} \operatorname{cotang} \alpha . \quad . \quad . \quad, \quad . \quad . \quad . \quad .
$$

und als Bestimmungsgleichung für $\vartheta_{1}$ :

$$
\pi \frac{l}{h}=\ln \frac{\sin \left(\vartheta_{1}-\delta\right)}{\sin \left(\vartheta_{1}+\delta\right)}-\left(n-2 \vartheta_{1}\right) \operatorname{tang} \alpha
$$

denn $l, h$ und $\delta=\frac{\pi}{2}-\alpha$ sind durch ein vorgelegtes Gitter bestimmt. Für $\vartheta_{2}=1 / 2 \pi$ wird $\frac{l}{h}$ gleich Null und für $\vartheta_{1}=\delta$ wird es negativ unendlich. Da $l$ infolge der Wahl des Koordinatensystems negativ ist, werden durch die Werte für $i_{1}$ von $1 / 2 \pi$ bis $\delta$ vorkommende Fälle beherrscht. $\theta_{1}<\delta$ ist aurgescblossen, weil sonst $\zeta_{0}$ oder $\zeta_{1}$ in die 
untere Halbebene fällt. $\vartheta_{1}>{ }_{2}^{\pi}$ liefert nach Vertauschung von $\zeta_{0}$ und $\zeta_{1}$ das gleiche Resultat wie oben.

Ferner folgt aus der unendlichen Vieldeutigkeit des Logarithmus, daß sich alle Periodenstreifen in gleicher Weise auf die obere $\zeta$-Halbebene abbilden.

Für die praktische Rechnung können folgende Näherungsformeln benutzt werden:

$$
\begin{aligned}
& \frac{l}{h} \text { klein: } 1-\frac{r_{0}}{r_{1}}=\frac{b}{t}\left[1-\frac{b}{2 t}+\left(\frac{b}{2}\right)^{2}\left(1+\frac{2 \cos 2 \alpha}{3 \cos ^{2} \alpha}\right)\right], \\
& \quad{ }_{h}^{l} \text { grob: }{ }_{r_{1}}^{r_{0}}=e^{2} a \tan a-\pi \frac{l}{h},
\end{aligned}
$$

wenn $b=\frac{l}{\cos \alpha}$ die $»$ Gittertiefe « und $t=\frac{h}{\cos \alpha}$ die Gitterteilung « ist. (Siehe Abb. 1)

4. Die Stromfunktion. Zur Stromfunktion gelangen wir, wenn wir fordern, daß die Strömung von $z=-\infty$ kommen soll, dort unter einem bestimmten Winkel gegen die $x$-Achse geneigt ist und nach $z=+\infty$ abfließen soll, mit der Bedingung, da $B$ an der Hinterkante der Schaufel die Strömungsgeschwindigkeit endlich bleibt. Durch die Abbildung sind die Pankte $\zeta_{0}$ and $\zeta_{1}$ gegeben. Legen wir in sie eine Wirbelquelle bezw. eine Wirbelsenke; damit die $\xi$-Achse Stromlinie wird, muß das Spiegelbild hjnzugenommen werden. Ist $Q$ das Maß für die Quellstärke und $S$ das Maß für die Senkstärke, so lautet der Ansatz:

$$
\Omega(\boldsymbol{z})=\Omega[f(\zeta)]=\omega(\zeta)=C Q\left[e^{-i \tau} \ln \left(\zeta-\zeta_{0}\right)+e^{i \sigma} \ln \left(\zeta-\zeta_{0}\right)\right]
$$

$$
+C S\left[e^{-i i} \ln \left(\zeta-\zeta_{1}\right)+t^{i \tau} \ln \left(\zeta-\zeta_{1}\right)\right] . \quad(15)
$$

wobei $\sigma$ und $\tau$ von der Normalen zur Gitterachse links herum positiv gezäblt werden. Die Geschwindigkeitskomponenten in der $x$-Richtung bezw. $y$-Richtung sind:

$$
\begin{array}{ll}
u-\infty=Q \cos (\alpha+\sigma) ; & u+\infty=S \cos (\alpha+\tau), \\
v-\infty=Q \sin (\alpha+\sigma) ; & v+\infty=S^{\gamma} \sin (\alpha+\tau) .
\end{array}
$$

Die Zirkulation um eine einzelne Schaufel bestimmt sich aus der Forderung der endlichen Geschwindigkeit an der Hinterkante der Schaufel. Ist die Zirkulation bekannt, so ist damit der Zusammenhang zwischen Anströmrichtung und Abflußrichtung im Unendlichea gegeben, also die Beziehung zwischen $Q, S, \sigma, \tau$.

Der Hinterkante entspricht in der $\zeta$-Ebene der Punkt $\zeta=\infty$. In ihm war $\frac{d z}{d \zeta} \sim c^{c}+\ldots$ Soll nun $\frac{d \Omega(z)}{d z}$ für $\zeta=\infty$ einen endlichen Weit erhalten, so muB $d \zeta(\xi)$ ebenfalls von der dritten Ordnung Null werden. Eine Reihenentwicklnng im Unendlichen ergibt:

$$
\begin{aligned}
& a_{\zeta}={ }_{5}^{2 O}(Q \cos \sigma+S \cos \tau)+{ }_{5}^{2 C}\left[2 r_{1} \cos \left(\theta_{0} \quad \sigma\right)+S r_{1} \cos \left(\theta_{1}-\tau\right)\right] \\
& +\frac{2}{\zeta^{3}}\left\{C r_{0}{ }^{2} \cos \left(2 \theta_{0}-\sigma\right)+S r_{1}^{2} \cos \left(2 \theta_{1}-\tau\right)\right\}+\ldots \ldots
\end{aligned}
$$

Damit nun das Nullwerden dritter Ordnung für $\breve{b}=\infty$ eintritt, müssen die Zähler der Glieder mit $\frac{1}{\zeta}$ und $\frac{1}{\xi^{2}}$ jedes für sich gleich Null werden. So ergeben sich die beiden folgenden Gleichungen:

und

$$
\begin{aligned}
& Q \cos \sigma=-S \cos \tau \text {. . . . . . . . (16) } \\
& Q r_{0} \cos \left(\theta_{0}-\sigma\right)=-N r_{1} \cos \left(\theta_{1}-\tau\right) . . . . . \quad \text {. (17). }
\end{aligned}
$$

Gl. (16) ist die Kontinuitätsbedingang, denn diejenige Flï ssigkeitsmenge, die seukrecht auf das Gitter znfliebt, muß auch in senkrechter Richtung abfließien.

Die durch die Abbildung gegebenen Größen sind $r_{0}, r_{1}, \vartheta_{0}, \vartheta_{1} ; Q$ und $\sigma$ sind durch das Anströmen bestimmt. Durch die beiden GL. (16) und (17) werden $S^{\gamma}$ und $\tau$ in $\mathrm{Ab}$ hängigkeit von diesen Größen festgelegt, $d$. h. für $z=+\infty$ die Strömungsgeschwindigkeit und die Stromrichtung. Mithin ist auch die Ablenkung, die die Strömung durch das Gitter erfthrt, bestimmt. Sie überträgt auf das Gitter eine Kraft, die nach zwei Komponenten zerlegt werden kann, und die mit Rücksicht auf die Anwendung auf die Turbinentheorie »Tangentialschub $T$ « und Axialschub $W$ heißen.

5. Tangentialschub und Axialschub. Von praktischem Interesse ist nun die Frage, wie bängen ZufluB- und Abflnbrichtang von der gegenseitigen Lage der Schaufeln $a b$, also von ibrer I,ănge, von ihrem gegenseitigen Abstande und von der Staffelung. Als 
Beziehung $z$ wischen Zufluß- und AbfluBrichtung ergibt sich mit Rücksicht auf oben angeführte Gleichungen:

$$
\tan g-\operatorname{tang} \tau=\left(1-\frac{r_{0}}{r_{1}}\right) \frac{\sin (\alpha+\sigma)}{\cos \alpha \cos \sigma} .
$$

Dieser Wert ist proportional dem Tangentialsehub $T$. Nennen wir $T_{\infty}$ den Wert den $T$ für den Grenziall $\frac{l}{h}=\infty$ annimmt, und bilden wir den Quotienten $\frac{T}{T_{\infty}}=1 \frac{r_{n}}{r_{1}}$, so gibt dieser Quotient an, wie groß für ein bestimmtes $\frac{l}{h}$ der Tangentialschab ist im Vergleich zu dem, der sich für den günstigsten Fall $\frac{l}{h}=\infty$ erreichen ließe. Aus Abb. 4 ist fïr den Sonderfall $\sigma=0^{0} \frac{T}{T_{\infty}}$ als Ordinate aufgetragen, ${ }_{t}^{b}$ als Abszisse. Um die Abhängigkeit von $\alpha$ zum Ausdruck za bringen, sind die Kurven fiir $\alpha=15^{\circ}, \alpha=30^{\circ}$ und $\alpha=45^{\circ}$ gezeichnet. Es zeigt sich, dab man in der Praxis gute Wasserbzw. Luftfïhrung auch durch kurze und weitstehende Schaufeln erreichen kann, was auch die neuesten Erfahrungen bestätigen. (Vergl. O esterlen, Schnelllanfende Wasserturbicen, Zeitschr. Ver. dentseh. Ing. Nr. 16, 1921.)

Andererseits läßt sich $T$ mit dem Auftrieb einer Einzelfläche $A$ vergleichen. Der Auftrieb einer Einzelflache ist nach Joukowski

$$
A=\frac{y}{g} \cup J
$$

$\left(\frac{\gamma}{g}=\right.$ Dichte, $U=$ Anströmgeschwindigkeit, $J=Z_{\text {Zir }}^{-}$ kulation). Aus der Zusammenfassung der oben angeführten Gleichungen folgt:

$$
\frac{T}{A}=\frac{\left(1-\frac{r_{0}}{r_{1}}\right) \frac{t}{b}}{\pi \cos \sigma}
$$

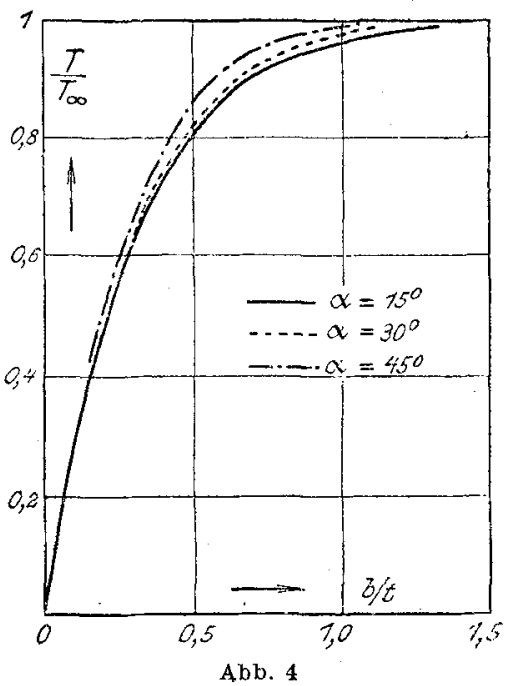

Der Faktor $\frac{1}{\cos \sigma}$ rührt von dem Richtungsunterschied von $T$ und $A$ her. Beschränken wir uns auf den Fall $\sigma=0$, so gilt für $\frac{b}{t}$ klein

$$
\frac{T}{A}=1-\frac{\pi \frac{b}{t}}{2}+\left(\frac{\pi \frac{b}{t}}{2}\right)^{2}\left(1+\frac{2 \cos 2 \alpha}{3 \cos ^{2} \alpha}\right) .
$$

Der Wert von $T$ nähert sich also bei Vergrößerung des Schaufelabstandes $(t=\infty)$ dem Werte $A, \mathbf{d}, \mathbf{h}$. die aufgestellte Formel geht für diesen Spezialfall in die Joukows ki sche für eine Einzelplatte über.

6. Gekrümmte Schaufeln und Schaufeln mit endlicher Dicke. Krummlinige Gitterprofile und Profile von endlicher Dicke werden analog erzeugt wie krummlinige Einzelprofile. Als Bild eines solchen Profils wählen wir in der $\zeta$-Ebene eine Gerade. Geht die Gerade durch den Nullpunkt and ist sie unter dem Winkel $\beta$ gegen die $\xi$-Achse geneigt, so ist ihr Bild eine unendlichdünne, gekrümmte Schaufel, deren Endtangenten wegen der Singularität in den Pankten $\zeta=0$ und $\zeta=\infty$ mit der $x$-Achse die Winkel $2 \beta$ bezw. $\pi-2 \beta$ einschließen. $\beta$ ist ein Maß für die Krümmung. Das Bild dicker Prolile in der $\zeta$-Ebene ist eine Gerade, die an dem Nullpunkt vorbeigeht. Die so gefundenen Profile stímmen mit den in der Praxis verwandten gut überein. Dicke Profile können wir aber anch bilden, wenn die Oberseite und Unterseite des Profils in der $\zeta$-Ebene durch Halbstrahlen gebildet werden, die im Nallpunkt um $k \pi$ gegeneinander geneigt sind, ganz in Analogie zu der in der Einleitung erwähnten Methode ').

1) Vergl r. Karman-Trefftz, a. a $O$ 
Die Stromfunktion für gekrümmte Profile erhalten wir ganz analog wie oben durch Spiegelung von $\zeta_{0}$ und $\zeta_{1}$ an der Geraden, die das Bild der Schaufel ist. Bei gekrümmten Profilen läßt sich neben glattem Abflus an der Hinterkante auch noch stoßfreier Eintritt erzielen. $\sigma$ und $\tau$ werden dann durch folgende Gleichungen bestimmt:

$$
\operatorname{tang} \sigma=\frac{\left(\frac{r_{0}}{r_{1}}+\frac{r_{1}}{r_{0}}\right) \cos \left(\vartheta_{1}+\beta\right)+2 \cos \left(\vartheta_{1}-\beta\right)}{\left(\frac{r_{0}}{r_{1}}-\frac{r_{1}}{r_{0}}\right) \sin \left(\vartheta_{1}+\beta\right)} ; \operatorname{tang} \tau=\frac{\left(\frac{r_{0}}{r_{1}}+\frac{r_{1}}{r_{0}}\right) \cos \left(\vartheta_{1}-\beta\right)+2 \cos \left(\vartheta_{1}+\beta\right)}{\left(\frac{r_{0}}{r_{1}}-\frac{r_{1}}{r_{0}}\right) \sin \left(\vartheta_{1}-\beta\right)}
$$

woraus nach Elimination von $\theta_{1}$ und $\frac{r_{0}}{r_{1}}$ folgt: $\sigma+\tau+2 \alpha=0$.

Für den Sonderfall ${ }_{h}^{l}=\infty$ ergibt sich bei gegebener $\Lambda$ blenkung $\tau-\sigma$ für $\beta$ ein

Minimalwert: $\beta_{\infty}=\frac{\tau-\sigma}{2}$

Diese Gleichungen lassen folgendes Resultat erkennen: StoBfreier Eintritt und glatter Abfluß werden also nicht erzielt, wenn die Strömung in großer Entfernung vor und hinter dem Gitter in Richtung der Schanfeltangenten filießt, sondern die Strömung ist gegen die Tangentenrichtung geneigt. Weicht die Strömung vor dem Gitter gegen die Tangentenrichtung nach rechts $a b$, so ist sie hinter dem Gitter um den gleichen Betrag nach links gerichtet.

7. Anwendung auf die Turbinentheorie. Es ist noch die Frage offen, wie die vorstehenden Betrachtungen auf die Turbinen zu übertragen sind. Bei Turbinen haben wir eine dreidimensionale Bewegung, wäbrend oben eine zweidimensionale behandelt ist. Es kann sich also bei der Uebertragung der Resultate nur um Näherungen handeln, die mehr oder weniger genau sind, entsprechend der Abweichvng der Flüssigkeitsbewegung in den Turbinen von einer ebenen Bewegang. Hierbei verhalten sich die einzelnen Turbinenarten verschieden. Zunächst sind auf ein feststehendes Leitwerk die obigen Resultate anwendbar. Für Axialturbinen, bei denen die Schaufelhöhe klein ist gegen den Durchmesser, so daß die Radialgeschwindigkeiten und ihre Ableitungen gegen die axialen und tangentialen Geschwindigkeiten vernachlässigt werden können, kann die Strömung ohne weiteres als eine ebene aufgefabt werden, wenn man sich die mittlere durch die Schaufei gelegte Zylinderfläche abgewickelt denkt. Um eine stationäre Strömung zu erbalten, beziebt man die Strömung durch das Leitrad auf ein festes Koordinaten yystem, die Strömung durch das Laufrad auf ein mitbewegtes, also in der $\Lambda$ bwicklung geradlinig gleichförmig bewegtes Koordinatensystem. In beiden Fällen hat man Strömungen durch Gitter, wie sie oben behandelt sind; die angegebenen Methoden sind also unverändert anwendbar.

Für Radialturbinen idealisiert man die Strömung in der Weise, daß man etwa eine Strömnng betrachtet, welche radial aus dem Unendlichen kommend von außen nach innen in ebener Strömung durch die rotationssymmetrisch angeordneten Leit- und Laufschaufeln strömt, um im Nullpunkt in einer Senke zu enden. Eine solche Strömung erhält man, wenn man die von uns behandelte Gitterströmung durch eine Transformation

$$
Z=\zeta^{1 / n} \text { (sithe G]. 1) }
$$

umformt, wobei aus der Parallelströmung durch das Gitter von unendlich vielen Schanfeln eine Radialströmung durch $n$ Schaufeln wird. Dies ist jedoch nur für die Strömung durch das Leitrad möglich; da man nämlich die Strömung durch das Lanfrad, om eine stationäre Strömung zu bekommen, auf ein rotierendes Koordinatensystem beziehen muß, ergibt sich jnbezug auf ein solches Koordinatensystem für das Potential die Differentialgleichung $J q=$ const, zu deren Integration zwar die Abbildung des Schaufelsystems auf die Ebene unverändert benutzt werden kann, die zu lösende Randwertaufgabe aber verändert wird, so dab auch die oben gegebenen Formeln für die auf die Schaufeln wirkenden Kräfte ihre Gültigkeit verlieren. Deshalb beschränken wir uns im folgenden auf Axialturbinen.

In den oben angestellten Betrachtungen handelt es sich immer um Relativströmung, d. h. es wird angenommen, daß das Gitter feststeht und die Flïssigkeit sich gegenüber dem Gitter bewegt. Fü̈r das Leitwerk lälst sich die Austrittsrichtung des Flüssigkeitsstromes also ohne weiteres angeben. Da nun aber das Laufrad sich gegenüber dem Leitwerk bewegt, ist nicht die Richtung eines jeden Flüssigkeitsfadens von Interesse, sondern ein Mittelwert, der sich aus den Richtnngen aller Stromfäden zusammenestzt. 
Die Schaufelform und Schaufelanordnung des Laufrades bestimmt die Relativströmung der Flüssigkeit zum Laufrade. Unter Berücksichtigung der absoluten Umfangsgeschwindigkeit des Laufrades $u$ und der Relativströmung der Flüssigkeit zum Laufrade, fï̈r die stoBfreier Eintritt gefordert wird, läBt sich die Absolutströmung $c_{a}$ ermitteln, wobei die Absolutströmung beim Laufradeintritt mit der Strömung beim Leitradaustritt übereinstimmen mub (Abb. 5). Durch diese Forderung ist die Stromrichlung beim Leitradaustritt bestimmt. Beim Austritt aus dem Laufrade wird die absolute Stromrichtang ebenso ermittelt wie beim Eintritt in das Lanfrad, wobei die Umfangsgeschwindigkeit des Laufrades dieselbe ist und die Richtung der Relativströmung mit der Richtung der Sohaufel-

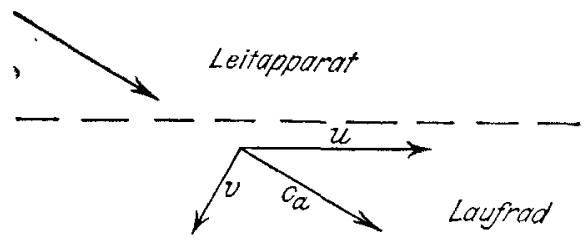

Abb. 5 tangente denselben Winkel einschließt wie beim Eintritt.

Die von dem Flüssigkeitsstrom auf das Laufrad übertragene Kraft zerlegen wir in zwei Komponenten, einmal den Tangentialschub und zum anderen in den Axialschub. Der Tangentialsohub wirkt in Richtung der Tangente an das Laufrad und ist für die Arbeitsleistung der Turbine bestimmend; er ist gleich der Impulsdifferenz beim Eintritt und Austritt der Flüssigkeit aus dem Laufrade, also dem aus der Relativströmung ermittelten Tangentialschub. Der Axialschub wird vom Lager aufgenommen oder hebt sich bei $\mathrm{Zwillingsanordnung} \mathrm{auf.} \mathrm{Er} \mathrm{bestimmt} \mathrm{sich} \mathrm{aus} \mathrm{der} \mathrm{Druckdifferenz} \mathrm{vor} \mathrm{und} \mathrm{hinter} \mathrm{dem}$ Laufrade in gleicher Weise wie bei der Relativströmung ${ }^{1}$ ).

\section{Über hyperboloidische Verzahnung.}

\section{Von E. STÛBBLER in Berlin-Dahlem.}

I seiner Abhandlung: Ueber die Verzahnung der Hyperboloidräder mit geradlinigem Eingrifi ${ }^{2}$ ) hat M Disteli diejenigen Raumverzahnungen behandelt, bei denen die Profillläehen, d. h. die Arbeitsflanken der Radzähne, windschiefe Regelflächen sind, die sich stets längs einer Erzengenden berïhren. Man wird vermuten, daß der Fall in die Ebene abwickelbarer Profilflächen als Sonderfall unter jenen Verzabnungen enthalten sei. Dies trifft aber merkwürdigerweise nicht zn. Der Grund liegt darin, da $\beta$ bei einer Bewegung zweier Regelflächen gegeneinander, wobei sie sich stets längs einer Erzeugenden berübren, die windschiefen Regelflächen sich ganz anders verhalten als die abwickelbaren. Wăhrend nämlich jene sich nur so berühren können, daß die Zentralpunkte der beiden koinzidierenden Erzeugenden, die ja auch denselben Verteilungsparameter ${ }^{3}$ ) besitzen mïssen, zusammenfallen, ist für die Berührung zweier abwickelbarer Flächen durchaus nicht Bedingung, daB die Zentralpunkte, die auf den Rückkehrkanten liegen, zusammentreffen.

So kommt es, daß der Fall abwickelbarer Profilflächen gesondert behandelt werden muß. Zudem sind doch diese Profilflächen leichter technisch herzustellen als die windschiefen, wenn man etwa die Schraubenflächen ausnimmt.

1) Vergl. Lr.-Thg, Bauersfeld, Die Grundlagen zur Berechnung schnellaufender Kreisel ruder, Zeit:ehr. Ver, deutseh. Ing. 19:2.

2) Zeitsebrift für Math. a. Phys. Bd. 59, 1911, S. 244.

3) Zur Erklärung dieser und einiger anderer hier verwendeter begritte aus der Geometrie der Regelfluchen sei folgendes gesig̨. Bewegt sich eincs Gerade im Raum so. dab zwei unmittelbar aufeinander folgende Lagen dinen Pukt geneinsam haben, so beschreil,t sie eino 》abwirkelbare" Regelflache. Eise »windschiete" Regelfiache entsteht, wenn zwei Nachbarlagen einer bewagten Geraden nieht nur dinen kleinen Winkel, condern wheh einen von Null versehiedenen kleinsten Abstand aufweisen.

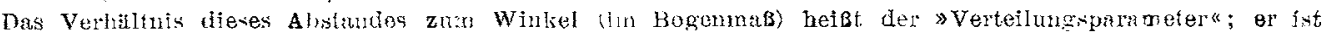
für die ubwickelbaren Regelf itehen Null. Der Zentralpunkt ist der Punkt der Geraden, weleher der Nachbargeraden an nachsten gelegen ist; eine Ebene durch die Gerade, welche zur Nachbargeraden parallel ist, heibt "asyinptotische* Ebene, die dazu senkrechte Ebene "Zentralebene*, das gemeinsame Lot von Nachbargeraden "Striktionsstrabl", der Ort der Zentralpunkte "Striktionslinie (bei der ab.

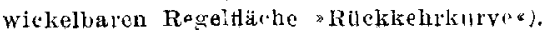

